\title{
Some methods for calculating and optimizing the characteristics of rods made of composite materials
}

\author{
Tatiana Bobyleva ${ }^{1, *}$ and Alexey Shamaev ${ }^{2,3}$ \\ ${ }^{1}$ Moscow State University of Civil Engineering, (NRU MGSU), Moscow, Russian Federation \\ ${ }^{2}$ Ishlinsky Institute for Problems in Mechanics of the Russian Academy of Sciences, Moscow, \\ Russian Federation \\ ${ }^{3}$ Lomonosov Moscow State University, Russian Federation
}

\begin{abstract}
Composite materials consisting of several phases are widely used in modern construction. Numerous experiments have shown that the properties of structurally heterogeneous materials can differ significantly from those of the individual components making up the composition. Besides, rapidly changing coefficients of differential equations describing such composite materials greatly complicate the solution of boundary value problems even with the help of computer calculation methods. Therefore, the homogenization method is used. In this paper the approach propose to obtain in explicit analytical form the effective model of the problem of loading a heterogeneous pipe made of layered material, provided that the elastic properties of the material only depend on the distance from the center of pipe cross section. We point to a method that obviously leads to an analytical result. It follows from the article that it is possible to choose the function that determines the structure of the "winding" in such a way as to obtain the stiffness characteristics of the pipe as close as possible to the desired with fixed mass fractions of the materials used.
\end{abstract}

\section{Introduction}

Problems for products from heterogeneous materials, in particular pipes, arise in many areas of construction. To improve the strength characteristics of pipes during their manufacture, several layers of various materials are used, which together provide high tightness and strength. Layers often vary considerably in stiffness. Examples include fibrous composites formed by longitudinal-transverse winding. Such heterogeneity is the cause of their specific behavior during deformation. The method of asymptotic averaging turns such a material into a homogeneous one, which is described by averaged equations. This averaging method is stated, for example in $[1,2]$, one of the first applications of which was the problems of the theory of elasticity [3, 4]. Several examples of its application are given in [5-8].

\footnotetext{
* Corresponding author: tatyana2211@outlook.com
} 
In this article we also want to consider the composite pipe from several materials. The load is applied in the form of the force field acting on the surface and distributed inside the volume. One of the tasks of this work is the replacement of a layered cylindrical tube with a cylindrical tube, which has elastic and elastic-creeping characteristics slowly depend on the radius and therefore large finite elements and large-pitch grids can be used to calculate stress-strain states. Averaged characteristics of stiffness and creep can be represented in explicit analytical form.

A similar problem was considered in [9-12]. In these articles it was not a cylindrical tube, but a rod with an arbitrary periodic structure. In the case under consideration, we can explicitly construct the above-mentioned effective characteristics effective characteristics of a one-dimensional rod and solve the loading problem analytically. This is due to the fact that in this case it is possible to explicitly solve the auxiliary boundary value problems on a periodicity cell necessary for constructing effective characteristics. These boundary value problems have periodic boundary conditions along the elastic rod and the corresponding Neumann conditions on the lateral surface. In this case, by introducing cylindrical coordinates, we can reduce these auxiliary three-dimensional problems of the theory of elasticity to one-dimensional systems, where only the radial variable is an independent variable (the angular and axial variables are not included in this system of equations). Systems of equations have a divergent form. As a result of such a structure, we can explicitly obtain an analytical solution. In this case, the condition of periodicity in the direction along the pipe is fulfilled automatically, since this solution doesn't depend on the axial coordinate. It is well known that the Neumann problem doesn't always have a solution. There are necessary and sufficient conditions for the existence of a solution connecting the boundary conditions with the right-hand side of the system. Auxiliary boundary value problems contain constant matrices. It is the choice of these constant matrices that will ensure that the solvability conditions are satisfied. The elements of these matrices will be the numbers that determine the effective characteristics of the heterogeneous pipe, namely, the effective indicators of flexural rigidity, tensile stiffness and torsion. Authors receive these effective indicators and auxiliary functions in an explicit analytical form. Forces applied to the surface can be selected in various ways. It can simulate a force load applied in a direction perpendicular to the pipe and parallel to the pipe, as well as torsion around an axis directed along the pipe or perpendicular to it. The available analytical solutions allow to evaluate qualitative and quantitative characteristics of obtained stress-strain states.

\section{Problem specification and decision}

Consider the system of equilibrium equations of the theory of elasticity [1] in Cartesian coordinates $x_{1}, x_{2}, x_{3}$

$$
\frac{\partial}{\partial x_{j}}\left(A^{i j}\left(\frac{\varphi(\vec{x})}{\varepsilon}\right) \frac{\partial \vec{u}}{\partial x_{i}}\right)=\vec{f}
$$

specified in a three-dimensional hollow cylinder (pipe)

$P \equiv\left\{r_{0}<x_{1}^{2}+x_{2}^{2}<R^{2}, \quad x_{3} \in(0 ; z]\right\},(i, j=1,2,3)$. 
The dependence of the matrices $A^{i j}(t)$ on the scalar variable $t$ is assumed to be a periodic, $\varphi=\varphi\left(x_{1}, x_{2}\right)$ is the smooth function, vector $\vec{u}$ is the displacement vector, $\vec{f}$ is the vector of mass forces, $\varepsilon$ is the small parameter, $\varepsilon \in(0 ; 1)$. The case of a layered cylinder with a periodic layer structure corresponds to the function $\varphi=\sqrt{x_{1}^{2}+x_{2}^{2}}$.

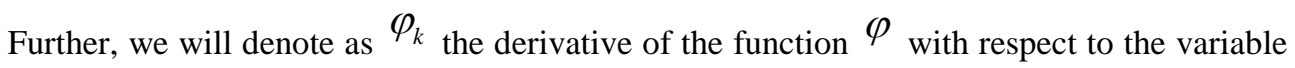
$x_{k}(k=1,2,3)$ and $y=\left(x_{1}, x_{2}, x_{3}\right)$.

We will define matrices

$$
\begin{gathered}
B_{\varepsilon}^{0}(t, y)=B^{0}\left(\frac{t}{\varepsilon}, y\right)=\left[\varphi_{l}(y) \varphi_{k}(y) A^{k l}\left(\frac{t}{\varepsilon}\right)\right]^{-1} \\
B_{\varepsilon}^{s}(t, y)=B^{s}\left(\frac{t}{\varepsilon}, y\right)=\left[\varphi_{l}(y) \varphi_{k}(y) A^{k l}\left(\frac{t}{\varepsilon}\right)\right]^{-1} \varphi_{p}(y) A^{p s}\left(\frac{t}{\varepsilon}\right) \\
B_{\varepsilon}^{i s}(t, y)=B^{i s}\left(\frac{t}{\varepsilon}, y\right)=\varphi_{j}(y) A^{k l}\left(\frac{t}{\varepsilon}\right)\left[\varphi_{l}(y) \varphi_{k}(y) A^{k l}\left(\frac{t}{\varepsilon}\right)\right]^{-1} \varphi_{p}(y) A^{p s}\left(\frac{t}{\varepsilon}\right)-A^{i s}\left(\frac{t}{\varepsilon}\right)
\end{gathered}
$$

In accordance with Theorem 7.13 [1], the set of matrices $\hat{A}^{i s}$ describing the "averaged" or "effective" properties of an elastic cylindrical body $P$ is given by the formulas

$$
\hat{A}^{i s}=\left\langle B^{i}\right\rangle^{*}\left\langle B^{0}\right\rangle^{-1}\left\langle B^{s}\right\rangle-\left\langle B^{i s}\right\rangle
$$

where $\left\langle B^{i}\right\rangle^{*}$ is the matrix conjugate to $\left\langle B^{i}\right\rangle$, and $\langle f\rangle$ means the period average of the function periodic in the variable $t$ :

$$
\langle f\rangle=\frac{1}{\tau} \int_{0}^{\tau} f(t) d t
$$
, $\tau$ is the period value.

It is obvious that with a given periodic configuration of the layers and given elastic characteristics of each of the materials included in the "winding", all the coefficients of the matrices $\hat{A}^{i s}$ can be calculated explicitly. The corresponding stress-strain state of the cylinder can be calculated as a solution of the following system

$$
\frac{\partial}{\partial x_{i}}\left(\hat{A}^{i s} \frac{\partial \hat{u}}{\partial x_{s}}\right)=\vec{f}(\vec{x})
$$

with the corresponding boundary conditions at the ends and the lateral surface of the cylinder $P$.

For our case of a circular section of a pipe the coefficients of the matrices $\hat{A}^{i s}$ will only depend on the next "slow" radial variable $r=\sqrt{x_{1}^{2}+x_{2}^{2}}$, and $x_{1}, x_{2}$. This arises due to the dependence of matrix coefficients on the derivatives $\varphi_{k}$ in the formulas $(2-4)$. The actual calculation of the stress-strain state of a "homogenized" cylinder can be done using, for example, by finite element method, which drastically reduces the amount of computation compared to the amount of computation needed to solve the original problem, 
which requires finite elements that are significantly smaller than width of each layer. In this case, we deal with calculations of the elastic deformed state of a three-dimensional body.

Now suppose that each of the phases of the composite material is an elastic-creeping material, and the relaxation kernels are given by the sums of a finite number of exponents which are decreasing functions of time $t$. We apply the Laplace transform in the time domain to the system (1)

$$
\tilde{f}(p)=\int_{0}^{\infty} f(t) e^{-p t} d t
$$

Then the system (1) takes the next form

$$
\frac{\partial}{\partial x_{j}}\left(\tilde{A}^{i j}\left(p, \frac{\varphi(\vec{x})}{\varepsilon}\right) \frac{\partial \tilde{u}}{\partial x_{i}}\right)=\tilde{f}(\vec{x}, p)
$$

For a fixed complex $p$, this system has the form (1), and when $\varepsilon \rightarrow 0$ the averaging method can be applied to its study. We get the formula (5) using this method. Therefore, for the Laplace transform formula (5) remains valid and the problem consists only in applying the inverse Laplace transform.

If the relaxation kernels are specified by a finite number of exponential functions, then as a result of the direct Laplace transform, the dependence on the variable $p$ will be fractionally rational. The calculation of matrix $B_{\varepsilon}^{0}, B_{\varepsilon}^{s}, B_{\varepsilon}^{i s}$ is performed by algebraic operations and that doesn't derive from the class of fractional rational functions. As a result of averaging by formula (5), we also don't leave this class, since averaging is carried out only by the radial variable and doesn't affect the dependence on $p$.

Thus, the matrix $\hat{A}^{i s}$ will consist of elements that are also fractionally rational functions of the variable $p$; therefore, the inverse Laplace transform will result in the sum of a finite (but larger than in the original problem) number of exponents. Explicit dependencies on "slow" variables $x_{1}, x_{2}$ will also be obtained.

\section{Summary}

The approach proposed in this paper proposes to obtain in explicit analytical form the solution of the problem of loading a non-uniform pipe made of laminated material, provided that the elastic- creeping properties of the material depend only on the distance from the center of pipe cross section. In the approach considered in this article, we do not go to the one-dimensional beam model, staying within the framework of the elastic or elastic-creeping body model. With the help of the finite element method our approach allows to calculate the picture of the stress-strain state of a cylindrical tube more accurately. The approach proposed in this paper permits one to obtain in an explicit analytical form the effective, slowly varying and radius-dependent elastic and elastic-creeping characteristics of a non-uniform pipe made of laminated material, provided that the elasticity of the material depends only on the distance from the center of the section of the pipe. In fact using the Laplace transform we can reduce the problem of determining creeping deformations under various long-term pipe loads to the problem considered in this article. In this case after the Laplace transform we obtain the problem of elastic equilibrium with coefficients depending on the complex parameter. Then, it will be necessary to perform the inverse Laplace transform which is possible when the initial creep (relaxation) kernels are sums of decreasing exponential functions. Inverse Laplace transform for such exponential kernels can be made explicit. 


\section{References}

1. O.A. Oleynik, G.A. Iosif'yan, A.S. Shamaev, Mathematical problems in elasticity and homogenization (Elsevier, North-Holland, 1992).

2. D.I. Bardzokas and A.I. Zobnin, Mathematical modeling of physical processes in composite materials of periodic structure (URSS, Moscow, 2005).

3. B.E. Pobedrya, Mechanics of composite materials (MSU, Moscow, 1984).

4. R.M. Christensen, Mechanics of composite materials (Dover, New York, 2005).

5. T.N. Bobyleva, Method of calculation of stresses in the layered elastic-creeping arrays (MATEC Web of Conf.), 86, 01024 (2016).

6. T.N. Bobyleva, A.S. Shamaev, Effective characteristics of a layered tube consisting of elastic creeping materials, MATEC Web of Conf., 251, 04039 (2018).

7. T.N. Bobyleva, A. S. Shamaev, An efficient algorithm for calculating rheological parameters of layered soil media composed from elastic-creeping materials, Soil Mechanics and Foundation Engineering, 54, 224 (2017).

8. T.N. Bobyleva, A.S. Shamaev, Method of approximate calculation of the stress tensor in layered elastic-creeping environments, IFAC-PapersOnLine, 51 (2), 138-143 (2018).

9. D. Lukkassen, A. Meidell, A. L. Piatnitski, A.S. Shamaev, Twisting a thin periodically perforated elastic rod, Applicable Analysis, 88 (10), 1563-1577 (2009).

10. D. Lukkassen, A. Meidell, A. L. Piatnitski, A.S. Shamaev, Symmetry-relations for elastically deformed periodic rod-structures, Math. Models and Methods in Applied Sciences, 19 (4), 501-525 (2009).

11. G. Panasenko, Asymptotic analysis of bar systems (I), Russian Journal of Math. Physics, 2 (3), 325-352 (1994).

12. G. Panasenko, Asymptotic analysis of bar systems (II), Russian Journal of Math. Physics, 4 (1), 87-116 (1996). 